\title{
Cullen sign in childhood malignancies
}

\author{
CC Au *, Karen KY Leung, KL Hon, Junita KY Tung, Carol LS Yan, WF Hui, WY Leung
}

Hong Kong Med J 2021;27:456.e1-2

https://doi.org/10.12809/hkmj219391

\section{Introduction}

We report four consecutive cases of young children with Cullen sign in a paediatric intensive care unit with abdominal malignancies and complications due to treatment. Two children had solid tumours with hepatic rupture and two had pancreatitis secondary to asparaginase use for leukaemia.

\section{Case 1}

A 23-month-old boy with ruptured liver metastasis and stage 4 neuroblastoma presented with abdominal distension due to a large retroperitoneal mass invading the liver and encasing the aorta and inferior vena cava. He required emergency laparotomy for haemorrhagic shock due to ruptured liver metastasis and endovascular embolisation of tumoural arteries. Subsequently there was an extensive subcutaneous haematoma around the surgical wound. Cullen sign was detected (Fig 1). Recurrent tumoural haemorrhage was excluded and surgical exploration performed to remove blood clots.

\section{Case 2}

A 5-year-old girl had asparaginase-associated pancreatitis and common B-cell acute lymphoblastic leukaemia. She was 6 months into her treatment protocol and received re-induction phase chemotherapy including pegaspargase (Oncaspar). She presented with abdominal pain and vomiting due to severe acute necrotising pancreatitis. Cullen sign (peri-umbilicus), Grey Turner sign (flank), and Fox sign (inguinal ligament) were detected (Fig 2). She

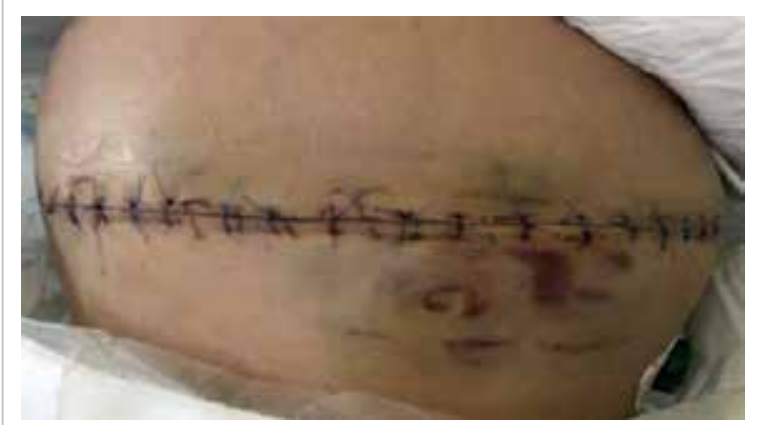

FIG I. 23-month-old boy who required emergency laparotomy for haemorrhagic shock due to ruptured liver metastasis and endovascular embolisation of tumoural arteries. Subsequently there was an extensive subcutaneous haematoma around the surgical wound. Cullen sign was detected required vasopressor and non-invasive ventilation for systemic inflammatory response syndrome. She later developed pancreatic pseudocyst and underwent ultrasound-guided drainage.

\section{Case 3}

A 5-year-old boy with asparaginase-associated pancreatitis and B-cell acute lymphoblastic leukaemia had received re-induction phase chemotherapy with pegaspargase (Oncaspar) and presented with abdominal pain and vomiting. He had severe acute necrotising pancreatitis and systemic inflammatory response syndrome. Cullen sign (peri-umbilicus) and Bryant sign (scrotum) were detected (Fig 3). He required vasopressor support. Later he developed multiple intrapancreatic and peripancreatic fluid collections and required repeated computed tomography-guided aspiration and drainage.

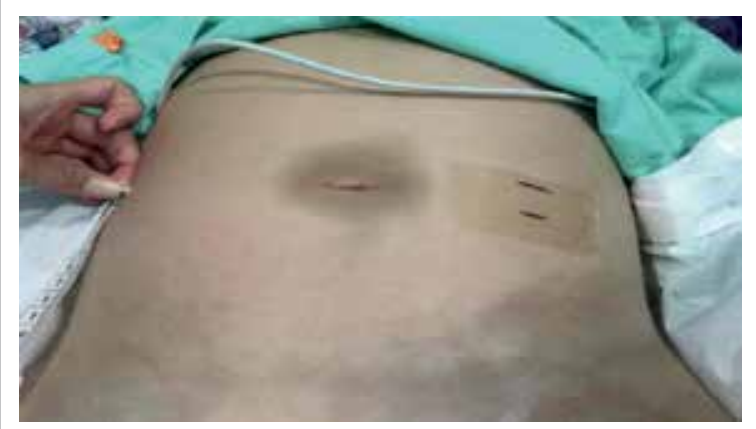

FIG 2. 5-year-old girl presented with abdominal pain and vomiting due to severe acute necrotising pancreatitis. Cullen sign (peri-umbilicus), Grey Turner sign (flank), and Fox sign (inguinal ligament) were detected

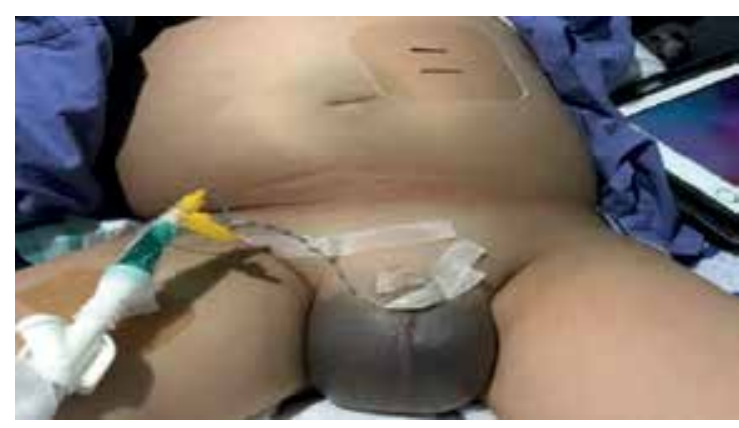

FIG 3. 5-year-old boy presented with abdominal pain and vomiting due to severe acute necrotising pancreatitis and systemic inflammatory response syndrome. Cullen sign (periumbilicus) and Bryant sign (scrotum) were detected 


\section{Case 4}

A 7-year-old boy with ruptured hepatoblastoma presented with abdominal distension. Hepatoblastoma involved all liver segments, extended into the inferior vena cava and had metastasised to the lungs. He required selective embolisation of left hepatic artery segments 2 and 3 for active haemorrhage. He had abdominal compartment syndrome and gross ascites that required drainage. Cullen sign was detected. (Fig 4) He was prescribed chemotherapy and consequently the tumour reduced in size.

\section{Discussion}

These four cases illustrate Cullen sign in paediatric malignancies. General practitioners and paediatricians should be aware of its diagnostic implications during clinical examination.

First described in 1918, Cullen sign referred to periumbilical ecchymosis due to retroperitoneal haemorrhage of ruptured ectopic pregnancy. ${ }^{1}$ It has become one of the classic abdominal signs in acute pancreatitis. Cullen sign or Grey Turner sign has been reported present in 3\% of acute necrotising pancreatitis cases. In 1984, these signs were reported to have a significant mortality rate of $37 \%,{ }^{2}$ but clinical outcomes of severe acute pancreatitis have since improved with intensive care. Nevertheless Cullen sign remains a pointer to serious intraabdominal haemorrhage. Haemorrhage can originate anywhere along an anatomical pathway. Pathophysiologically, blood tracks from the retroperitoneum through the gastrohepatic ligament to the falciform ligament of the liver, then reaches

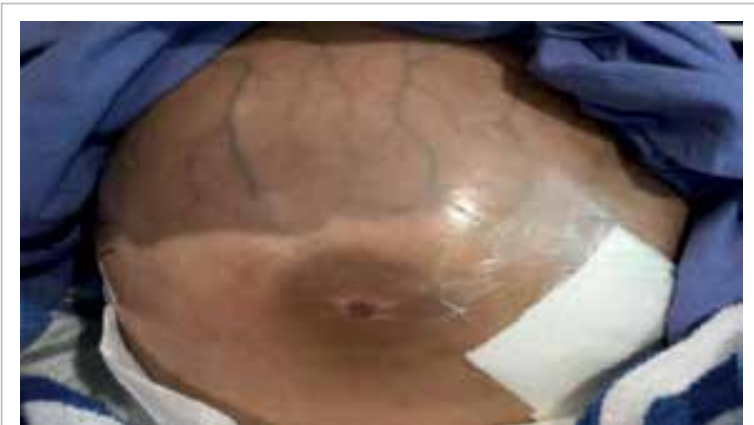

FIG 4. 7-year-old boy with abdominal compartment syndrome and gross ascites that required drainage. Cullen sign was detected the umbilicus through the round ligament of liver to form Cullen sign. ${ }^{3}$ Cullen sign has been reported in intra-abdominal malignancies, liver cirrhosis, and rectus sheath haematoma. ${ }^{4,5}$

\section{Author contributions}

All authors contributed to the concept or design of the study, interpretation of the data, drafting of the manuscript, and critical revision of the manuscript for important intellectual content. All authors had full access to the data, contributed to the study, approved the final version for publication, and take responsibility for its accuracy and integrity.

\section{Conflicts of interest}

As an editor of the journal, KL Hon was not involved in the peer review process for this article. Other authors have no conflicts of interest to disclose.

\section{Funding/support}

This study received no specific grant from any funding agency in the public, commercial, or not-for-profit sectors.

\section{Ethics approval}

The patients were treated in accordance with the Declaration of Helsinki. This study was approved by the Hong Kong Children's Hospital Research Ethics Committee (Ref HKCHREC-2019-009). Patients' parents consented for publication of clinical photographs.

${ }^{1} \mathrm{CC} \mathrm{Au}$ *, MB, BS, MRCPCH

${ }^{1} \mathrm{KKY}$ Leung, MB, BS, MRCPCH

${ }^{1} \mathrm{KL}$ Hon, MB, BS, MD

2 JKY Tung

${ }^{1}$ CLS Yan, MB, BS, MRCPCH

${ }^{1}$ WF Hui, MBChB, MRCPCH

${ }^{3}$ WY Leung, MB, ChB, FRCSEd(Paed)

Department of Paediatrics and Adolescent Medicine, The Hong Kong Children's Hospital, Hong Kong

2 Faculty of Medicine, The Chinese University of Hong Kong, Hong Kong

${ }^{3}$ Department of Surgery, The Hong Kong Children's Hospital, Hong Kong

* Corresponding author: aucc@ymail.com

\section{References}

1. Cullen TS. A new sign in ruptured extrauterine pregnancy. Am J Obstet Gynecol 1918;78:457.

2. Dickson AP, Imrie CW. The incidence and prognosis of body wall ecchymosis in acute pancreatitis. Surg Gynecol Obstet 1984;159:343-7.

3. Bem J, Bradley EL 3rd. Subcutaneous manifestations of severe acute pancreatitis. Pancreas 1998;16:551-5.

4. Mabin TA, Gelfand M. Cullen's sign, a feature in liver disease. Br Med J 1974;1:493-4.

5. Harris S, Naina HV. Cullen's sign revisited. Am J Med 2008;121:682-3. 